\title{
REVISTAMATERIA
}

ISSN 1517-7076 artigo 11544, pp.159-170, 2014

\section{Estudio estructural de arcillas de Chulucanas por difracción de rayos-X y método de Rietveld}

\author{
Structural study of Chulucanas clays by \\ X-ray diffraction and Rietveld method
}

Elvira L. Zeballos-Velásquez ${ }^{1}$, Patricia C. Melero ${ }^{1}$, Alejandro L. Trujillo ${ }^{2}$, Mirian E. Mejía ${ }^{3}$, Mario Ceroni ${ }^{4}$

\author{
${ }^{1}$ Laboratorio de Cristalografía de Rayos X, Facultad de Ciencias Físicas. \\ ${ }^{2}$ Laboratorio de Arqueometría, Facultad de Ciencias Físicas. \\ ${ }^{3}$ Facultad de Química e Ingeniería Química. \\ ${ }^{4}$ Universidad Nacional Mayor de San Marcos. Ap. Postal 14-0149. Lima, Perú. \\ e-mail: cristalografiarx@gmail.com, ezeballosv@unmsm.edu.pe
}

\section{RESUMEN}

Resultados de medidas de difracción de rayos-X mostraron una secuencia de modificaciones químicas y estructurales como la deshidratación, descomposición y formación de nuevas fases en arcillas sometidas a aumentos graduales de temperatura. Los componentes de las muestras fueron determinados, identificándose montmorillonita-Ca, vermiculita, muscovita, caolinita, chlorita, illita, cuarzo y albita. El refinamiento estructural por el método de Rietveld reportó, en todas las muestras, mayor porcentaje de arcilla noexpansiva en comparación con la expansiva. A $250^{\circ} \mathrm{C}$, la montmorillonita-Ca presentó una disminución drástica en su distancia interlaminar, de $1.5 \mathrm{~nm}$ a $1.0 \mathrm{~nm}$, debido a la remoción del agua superficial y de los poros, así como de la contenida en la región interlaminar. Para el caso de la vermiculita, esta disminución es mucho menor $(\sim 0.04 \mathrm{~nm})$, en tanto que la caolinita no presenta disminución significativa de este parámetro. La vermiculita estructuralmente colapsa a $\sim 350^{\circ} \mathrm{C}$, en tanto que la caolinita lo hace a $\sim 500^{\circ} \mathrm{C}$, mientras que nuevas fases aparecen, como la ortoclasa y anorthita.

Palabras llave: arcilla, difracción de rayos-X, método de Rietveld.

\section{ABSTRACT}

Results of measures of X-ray diffraction showed a sequence of chemical and structural changes such as dehydration, decomposition and formation of new phases in clays subjected to gradual increases in temperature. The components of the samples were determined identifying Ca-montmorillonite, vermiculite, muscovite, kaolinite, chlorita, illite, quartz and albite. The structural refinement by the Rietveld method reported the presence of a higher percentage of non-expansive clay compared to the expansive in all samples. At $250^{\circ} \mathrm{C}$, Ca-montmorillonite presents a drastic decrease in its interlayer distance, from $1.5 \mathrm{~nm}$ to $1.0 \mathrm{~nm}$, due to removal of water from the surface and pores, as well as of water contained in the interlayer region. In the case of vermiculite, this decrease is much smaller $(\sim 0.04 \mathrm{~nm})$, while kaolinite does not present significant decrease in this parameter. Vermiculite structurally collapses to $\sim 350^{\circ} \mathrm{C}$, whereas kaolinite makes it to $\sim 500^{\circ}$ $\mathrm{C}$, while new phases appear, such as orthoclase and anorthite.

Keywords: clay, X-ray diffraction, Rietveld method.

\section{INTRODUCCIÓN}

Las arcillas, del grupo de los filosilicatos, presentan estructura laminar de dimensiones nanométricas, constituida por planos de iones oxígeno e hidroxilos. Algunas de ellas (esmectitas y arcillas expansivas) pueden albergar cationes hidratados y agua en su interlámina, provocando un aumento del espaciado reticular y consecuentemente el hinchamiento del sistema, como en el caso de la montmorillonita. Este proceso depende 
del balance entre la atracción electrostática catión-lámina y la energía de hidratación del catión [1]]. A medida que se intercalan capas de agua y la separación entre las láminas aumenta, las fuerzas que predominan son de repulsión electrostática entre láminas, lo que contribuye a que el proceso de hinchamiento pueda llegar a disociar completamente unas láminas de otras. Cuando el catión interlaminar es $\mathrm{Na}$, las esmectitas tienen una gran capacidad de hinchamiento, pudiendo llegar a producirse la completa disociación de cristales individuales, con un alto grado de dispersión y un máximo desarrollo de propiedades coloidales. Si los cationes son de Ca o Mg, el hinchamiento es más reducido [2]

La estructura de las arcillas influye en sus propiedades físicas, por lo que es importante entender los cambios estructurales producidos por la temperatura durante la cocción de estos materiales en la producción de cerámicos [3] . En el presente trabajo, y tomando en cuenta resultados de trabajos anteriores [4, 9$]$, analizamos los cambios estructurales relativos a la región interlaminar en muestras de la región de Chulucanas, en función de los parámetros de temperatura y tiempo de tratamiento térmico, a fin de correlacionarlos con las propiedades. La evolución de las fases en cada muestra, como consecuencia del tratamiento térmico, es acompañada por medidas de Difracción de Rayos-X (DRX) luego de cada tratamiento. El análisis de la reflectividad es realizado por refinamiento estructural, aplicando el Método de Rietveld mediante el programa TOPAS. En trabajo paralelo [10] presentamos resultados del estudio de las muestras por Espectroscopia Mössbauer y Fluorescencia de Rayos-X.

\subsection{Refinamiento de la estructura por el método de Rietveld}

Las arcillas, particularmente las expansivas como la montmorillonita, representan un desafío en el refinamiento estructural debido a sus estructuras estratificadas. La naturaleza semi-cristalina de estos materiales, derivada del desorden asociado con las estructuras estratificadas, produce perfiles de difracción ampliamente asimétricos, con "backgrounds" complicados, que pueden ser difíciles de interpretar. Una alternativa para el análisis de estos perfiles es el uso del Método de Rietveld, el cual permite evaluar el proceso de transformación de la arcilla desde su estado en el medio ambiente, su contracción luego de ser sometida a la cocción durante la producción de cerámicos, hasta el colapso de su estructura por efecto del aumento de temperatura $[\underline{11}, \underline{12}]$. En muestras multifásicas, el método también proporciona información cuantitativa del porcentaje en peso de las fases presentes en la muestras.

El Método de Rietveld utiliza estructuras atómicas conocidas para generar un modelo teórico inicial de la estructura de las fases presentes en la muestra, por lo que éstas deben ser previamente identificadas. A partir de este modelo inicial, el método permite refinar los parámetros estructurales, en base al análisis de mínimos cuadrados, hasta que el modelo concuerde con el perfil experimental Este método también puede producir resultados cuantitativos precisos y reproducibles, así como compensar la intensidad inexacta de un pico de difracción debido a la orientación preferencial $[\underline{13}, \underline{15}]$.

Información sobre estructuras atómicas de los tipos básicos de arcillas están disponibles en la literatura, particularmente para las no-expansivas. Sin embargo, muy pocas estructuras de arcillas expansivas han sido caracterizadas y no se cuenta con suficiente información. Esto se debe, en parte, a que estas estructuras son inestables debido a sus propiedades de intercambio de cationes y absorción de agua, lo que dificulta una cristalización adecuada para su determinación estructural por rayos-X.

En el modelo teórico se incluye aspectos estructurales tales como: estructura cristalina, grupo espacial, posición de los átomos en la celda unitaria, etc. Asimismo, intervienen factores microestructurales que contemplan la concentración de las fases presentes, tamaño de cristal y microdeformaciones; también incluye factores instrumentales como el efecto de la óptica del equipo de DRX sobre el ancho de los picos de difracción.

La reflectividad calculada es obtenida por la introducción directa de diversos datos cristalográficos proporcionados por la literatura. La intensidad $y_{c i}$ de la reflectividad calculada está determinada por la suma de las contribuciones de las reflexiones vecinas para todas las fases $p$ en una muestra multifásica, más el incremento del background $b$, según la siguiente relación

$\left.y_{c i}=\left.S_{R}\left|\sum_{p} s_{p} \underline{A b}\right| \sum_{K}|| F_{K}\right|^{2} \Phi\left(2 \theta_{i}-2 \theta_{K}\right) \underline{A s} L_{K} P_{K} \|_{p}+y_{b i}\right\rfloor$

donde

$s_{p}:$ factor de escala para la fase $p$

$S_{R}$ : modelo de la función para los efectos de rugosidad superficial 
$\underline{A b}$ : factor de absorción

$F_{K}:$ factor de estructura

$\Phi: \quad$ función de modelamiento de los picos de reflexión

As : función de asimetría

$L_{K}$ : función de Lorentz-polarización y multiplicidad

$P_{K}: \quad$ función de orientación preferencial

$y_{b i}: \quad$ contribución del background

Los valores de estos parámetros son ajustados durante el refinamiento para cada fase, a fin de alcanzar la mejor aproximación entre el perfil calculado y el observado, sin perjuicio de la calidad del refinamiento de los parámetros. Este ajuste es realizado mediante minimización del residual $S$ por mínimos cuadrados según la relación

$$
S_{y}=\sum_{i} w_{i}\left(y_{i}-y_{c a l}\right)^{2}
$$

donde

$W_{i}: 1 / y i$,

$y_{i}: \quad$ intensidad observada en el $i$-ésimo paso

$y_{c a l}: \quad$ intensidad calculada en el $i$-ésimo paso

En el caso de muestras cristalinas o con contenido amorfo despreciable, el método permite calcular la cantidad en peso de cada fase presente en la muestra, a partir del factor de escala de cada fase, según la relación

$$
W_{p}=\frac{S_{p}(Z M V)_{p}}{\sum_{i=1}^{N} S_{i}(Z M V)_{i}}
$$

donde

$p$ : valor de la $i$-ésima fase de $N$ fases presentes en la muestra

$S_{i}:$ factor de escala

$Z M$ : peso de los átomos en la celda unitaria

$V$ : volumen de la celda unitaria

El análisis con el método de Rietveld provee numerosas ventajas sobre otros métodos de análisis cuantitativo, ya que el algoritmo modela el perfil. El uso del método ha permitido mejorar la exactitud de los resultados cuantitativos y extenderlo a sistemas complejos, como las arcillas, transformándose en una herramienta significativa para la investigación de estos materiales. En este trabajo usamos el programa TOPAS de Bruker-AXS [16,17], diseñado para el refinamiento de estructuras cristalinas basado en el Método de Rietveld.

\section{MATERIALES Y MÉTODOS}

Las muestras investigadas, provenientes de la región de Chulucanas (Piura) (Fig. 1) y preparadas por la Universidad de Piura, fueron proporcionadas por el Maestro Sr. Gerásimo Sosa Alache, ceramista de prestigio internacional. La Universidad de Piura prepara la arcilla en sus laboratorios (por sedimentación) y provee de este material a los artesanos de la región que lo solicitan, quienes la usan como materia prima para fabricar sus cerámicos, aunque la tendencia sigue siendo la preparación en los talleres artesanales. 


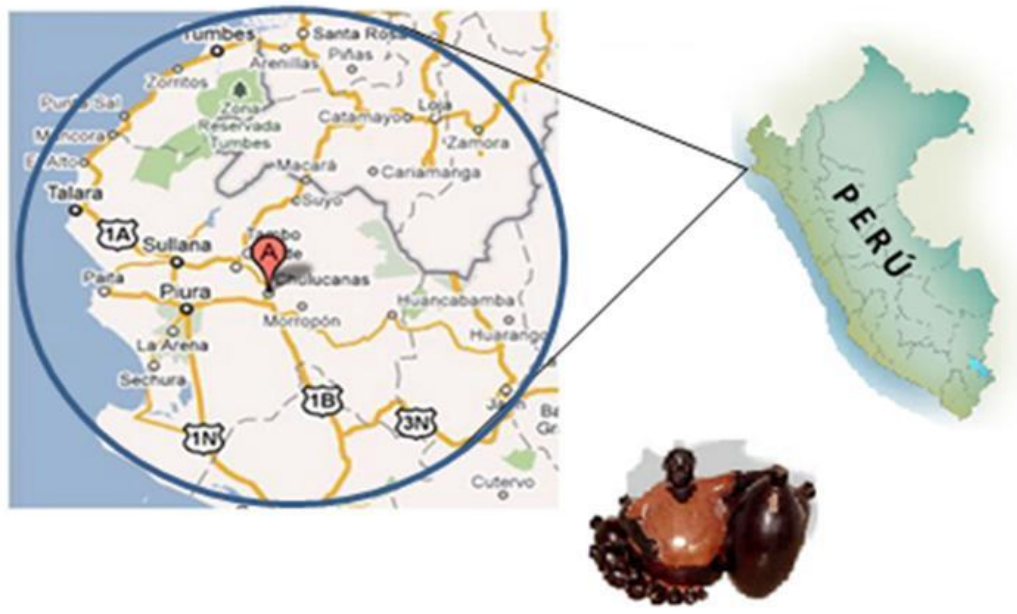

Figura 1: Región de Chulucanas (Piura), al norte de Perú. En la parte inferior, cerámica de Chulucanas de Gerásimo Sosa [18]..

Sin contar con información sobre la sedimentación de la muestra, ésta fue preparada para el proceso de cocción. La muestra (Fig.2), con aspecto de masa húmeda, fue secada en una mufla a $60{ }^{\circ} \mathrm{C}$, luego pulverizada con mortero y tamizada a $160 \mu \mathrm{m}$ tamaño de partícula.
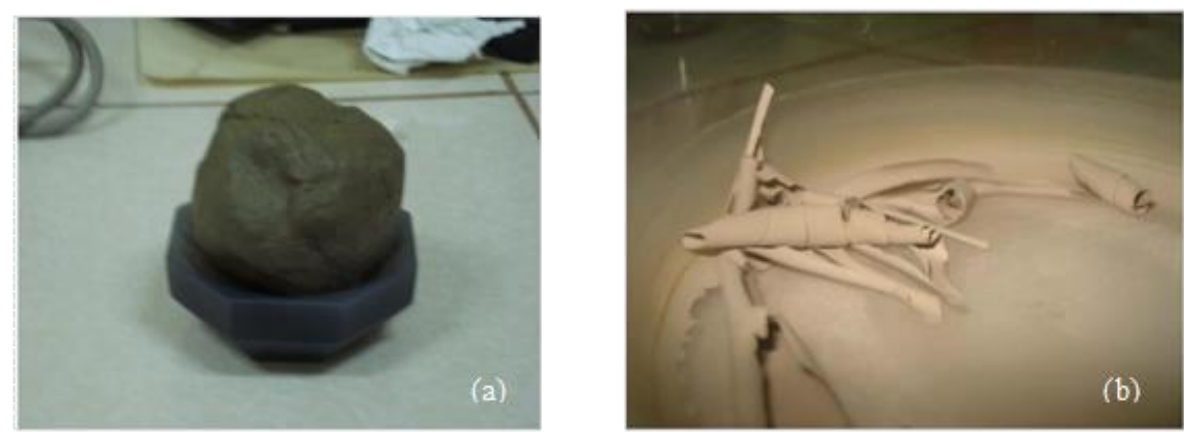

Figura 2: (a) Muestra húmeda de arcilla; (b) Muestra de arcilla luego de secada a $60{ }^{\circ} \mathrm{C}$.

El tratamiento térmico fue realizado utilizando un horno de tres etapas, Modelo PT 1600, en ambiente de aire. Las muestras fueron tratadas a temperaturas desde $100^{\circ} \mathrm{C}$ hasta $400^{\circ} \mathrm{C}$, con intervalos de $50^{\circ} \mathrm{C}$, y desde $400^{\circ} \mathrm{C}$ hasta $900^{\circ} \mathrm{C}$, con intervalos de $100^{\circ} \mathrm{C}$. En cada caso el tiempo de tratamiento fue de 2 hs. a temperatura constante, con una velocidad de ascenso de $5^{\circ} \mathrm{C} / \mathrm{min}$. Para cada temperatura fue usada una nueva muestra. La nomenclatura de las muestras está asociada a la temperatura de tratamiento (por ejemplo, R-ST: muestra sin tratamiento térmico; R-100: muestra a $100^{\circ} \mathrm{C}$, etc.). Medidas de DRX fueron realizadas antes y después de cada tratamiento térmico.

\section{RESULTADOS Y DISCUSIÓN}

Para las medidas de difracción de rayos-X utilizamos un difractómetro Bruker, modelo D8-Focus, geometría $\theta-2 \theta$, con radiación de $\mathrm{Cu}\left(\mathrm{K}_{\alpha}\right)$, "slit" receptor de $0.1 \mathrm{~mm}$, "slit" de divergencia de $1 \mathrm{~mm}$, y "soller" de $2.5^{\circ}$, con detector de centelleo; fue usado un voltaje de $40 \mathrm{kV}$ y corriente de $40 \mathrm{~mA}$. Las medidas fueron realizadas en el intervalo de $4^{\circ}$ a $65^{\circ}(2 \theta)$, con paso de $0.02^{\circ}$ y 4 s por paso. La (Fig. 3) muestra los difractogramas correspondientes a medidas antes del tratamiento térmico y con tratamiento de $100^{\circ} \mathrm{C}$ a $400^{\circ} \mathrm{C}$, con intervalos de $50^{\circ} \mathrm{C}$. 


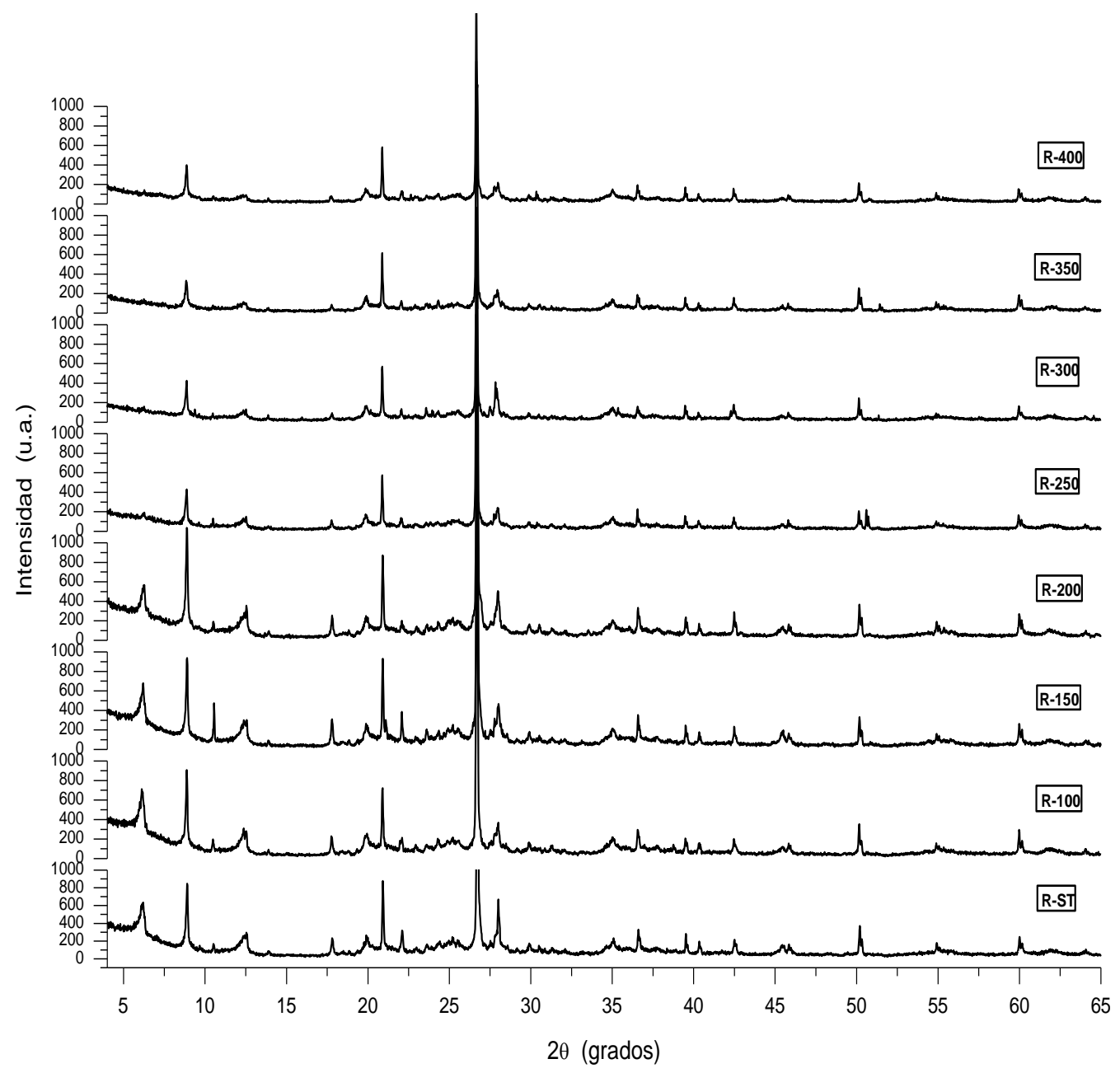

Figura 3: Difractogramas de muestras sin tratamiento térmico y con tratamiento de $100^{\circ} \mathrm{C}$ a $400^{\circ} \mathrm{C}$.

La identificación de fases fue realizada con el programa EVA de Bruker-AXS, determinándose la presencia de montmorillonita-Ca, vermiculita, muscovita, caolinita, chlorita, illita, cuarzo y albita, cuyos picos principales son indicados en la Figura 4. Estos resultados fueron confirmados por refinamiento estructural con el método de Rietveld, como es mostrado más adelante. Complementariamente, las muestras fueron evaluadas por Espectroscopia Mössbauer, cuyos resultados reportaron la formación de óxidos de hierro y la disminución de la proporción del sitio de $\mathrm{Fe}^{2+}$ asociado a la montmorillonita-Ca ("Evaluación del efecto de la temperatura en arcillas de Chulucanas usando Espectroscopia Mössbauer y Fluorescencia de Rayos-X”. Trujillo, A.L. et.al. Pendiente de publicación.) 


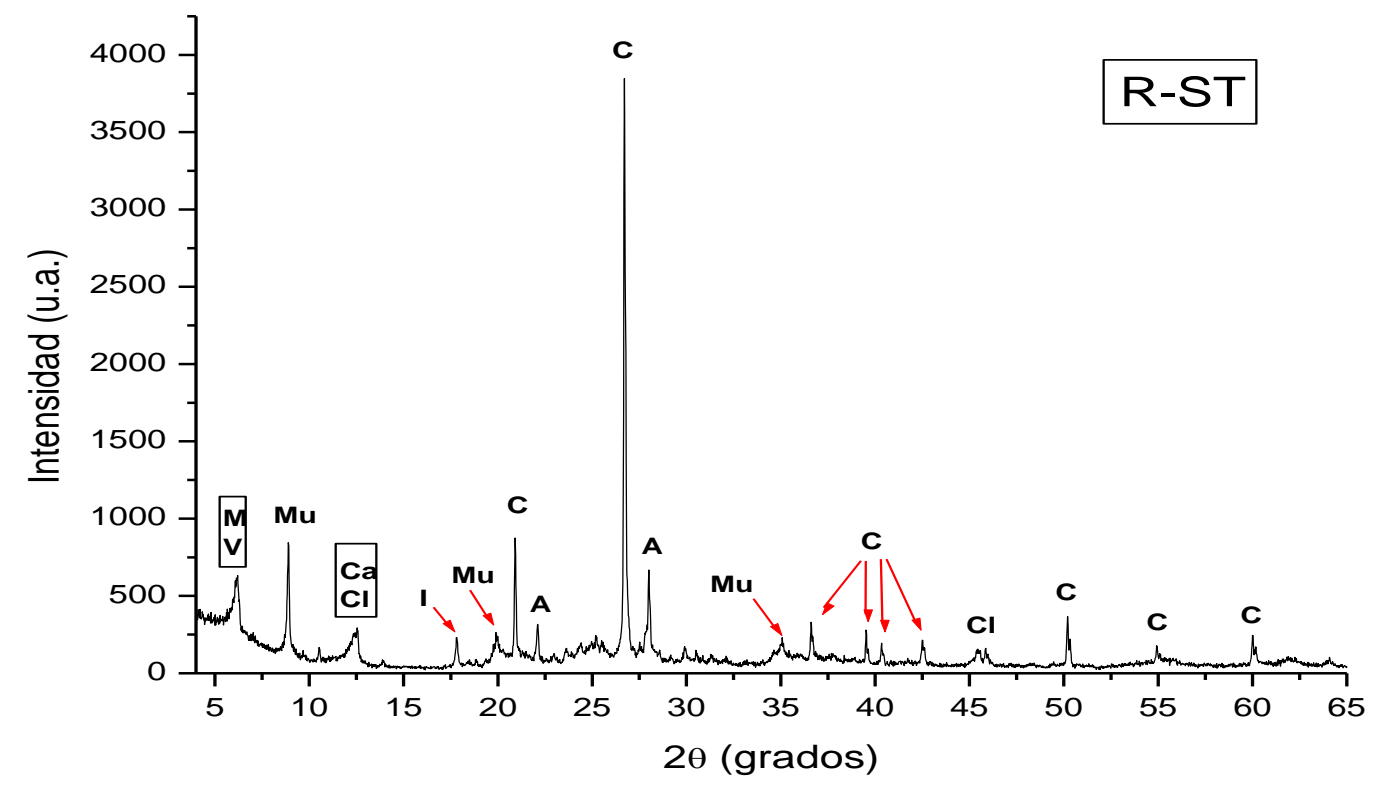

Figura 4: Difractograma de la muestra sin tratamiento térmico, con identificación de fases. (M: montmorillonita-Ca - PDF 13-0135; V: vermiculita - ICSD 034812; Mu: muscovita - ICSD 068548; Ca: caolinita - ICSD 087771; Cl: chlorita - ICSD 156166; I: illita - ICSD 090144; C: cuarzo - ICSD 027826; A: albita - ICSD 087654).

En la Figura 5 presentamos una vista ampliada de las medidas en el intervalo de $4^{\circ}$ a $10^{\circ}(2 \theta)$. Puede observarse que en los primeros calentamientos, la intensidad del pico (001) de la montmorillonita-Ca disminuye hasta desaparecer a la temperatura de $250^{\circ} \mathrm{C}$, lo que estaría indicando cambios estructurales en esta fase debido al aumento de la temperatura. Estos cambios están referidos particularmente a una disminución en su distancia interlaminar y, por lo tanto, un cambio de posición angular del pico (001), comportamiento característico de las arcillas expansivas. Las variaciones de este parámetro fueron calculadas por refinamiento estructural.

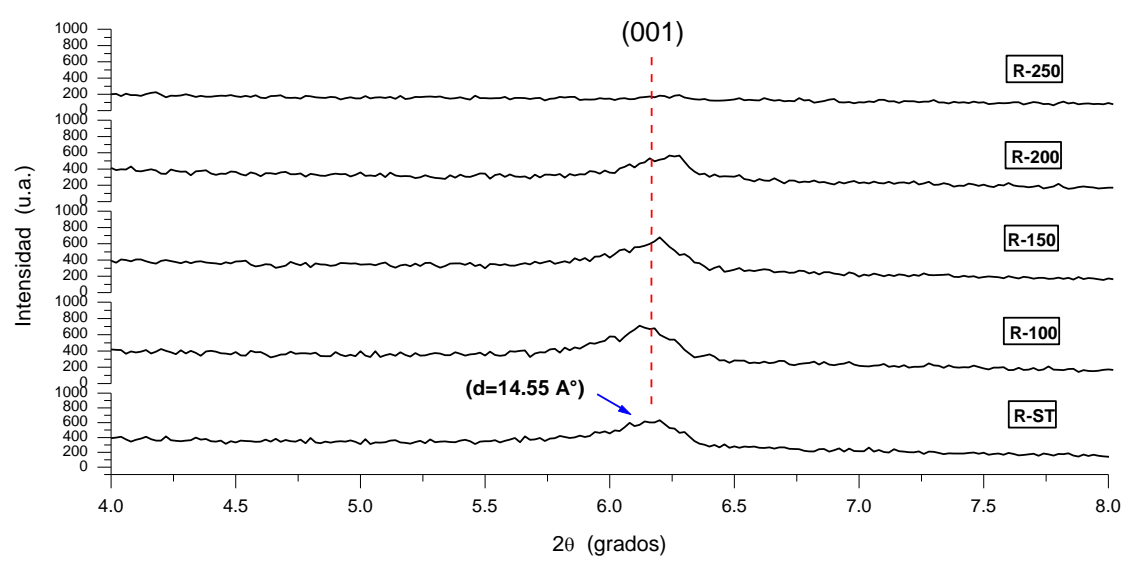

Figura 5: Decaimiento de la intensidad del pico (001) de la montmorillonita-Ca con el aumento de la temperatura. 
La Figura 6 muestra los difractogramas correspondientes a medidas después del tratamiento de $500^{\circ} \mathrm{C}$ a $900^{\circ} \mathrm{C}$, con intervalos de $100^{\circ} \mathrm{C}$.

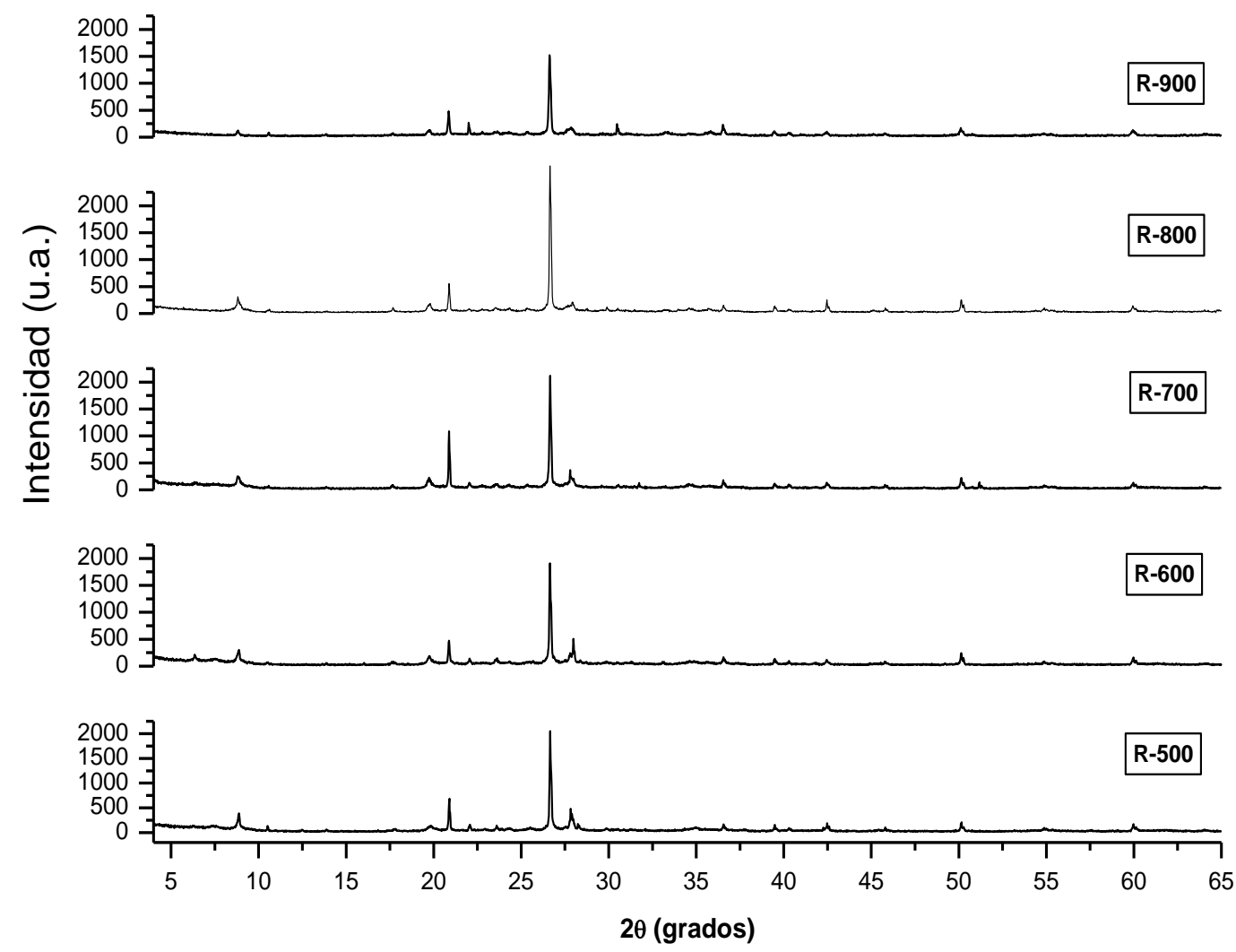

Figura 6: Difractogramas de muestras con tratamiento térmico de $500^{\circ} \mathrm{C}$ a $900^{\circ} \mathrm{C}$.

De la evaluación de los difractogramos observamos que las fases de vermiculita y caolinita desaparecen a temperaturas superiores a $300^{\circ} \mathrm{C}$ y $400^{\circ} \mathrm{C}$, respectivamente, como consecuencia del colapso de sus estructuras con el aumento de la temperatura; simultáneamente surgen nuevas fases, entre las cuales se estima la presencia de anorthita (ICSD 086330) a partir de $500^{\circ} \mathrm{C}$; orthoclasa (ICSD 081137) es identificada en muestras de menor temperatura (a partir de $200^{\circ} \mathrm{C}$ ).

Los parámetros estructurales y los porcentajes en peso de todas las fases, para el conjunto de muestras investigadas, fueron determinados por refinamiento estructural. Para el ajuste fue aplicada la función PVTCHZ (pseudo-Voigt Thompson-Cox-Hastings). En la Figura 7 presentamos resultados del refinamiento para las muestras sin tratamiento térmico y las tratadas hasta $300^{\circ} \mathrm{C}$; en La Figura 8 mostramos resultados del refinamiento para las muestras tratadas a temperaturas de 350 a $900^{\circ} \mathrm{C}$. No fue observada orientación preferencial. 
ZEBALLOS-VELASQUÉZ, El vira.; MELERO, Patricia.;TRUJILLO, Alejandro L,. revista Matéria, v.19, n.2, pp. 159-170, 2014.

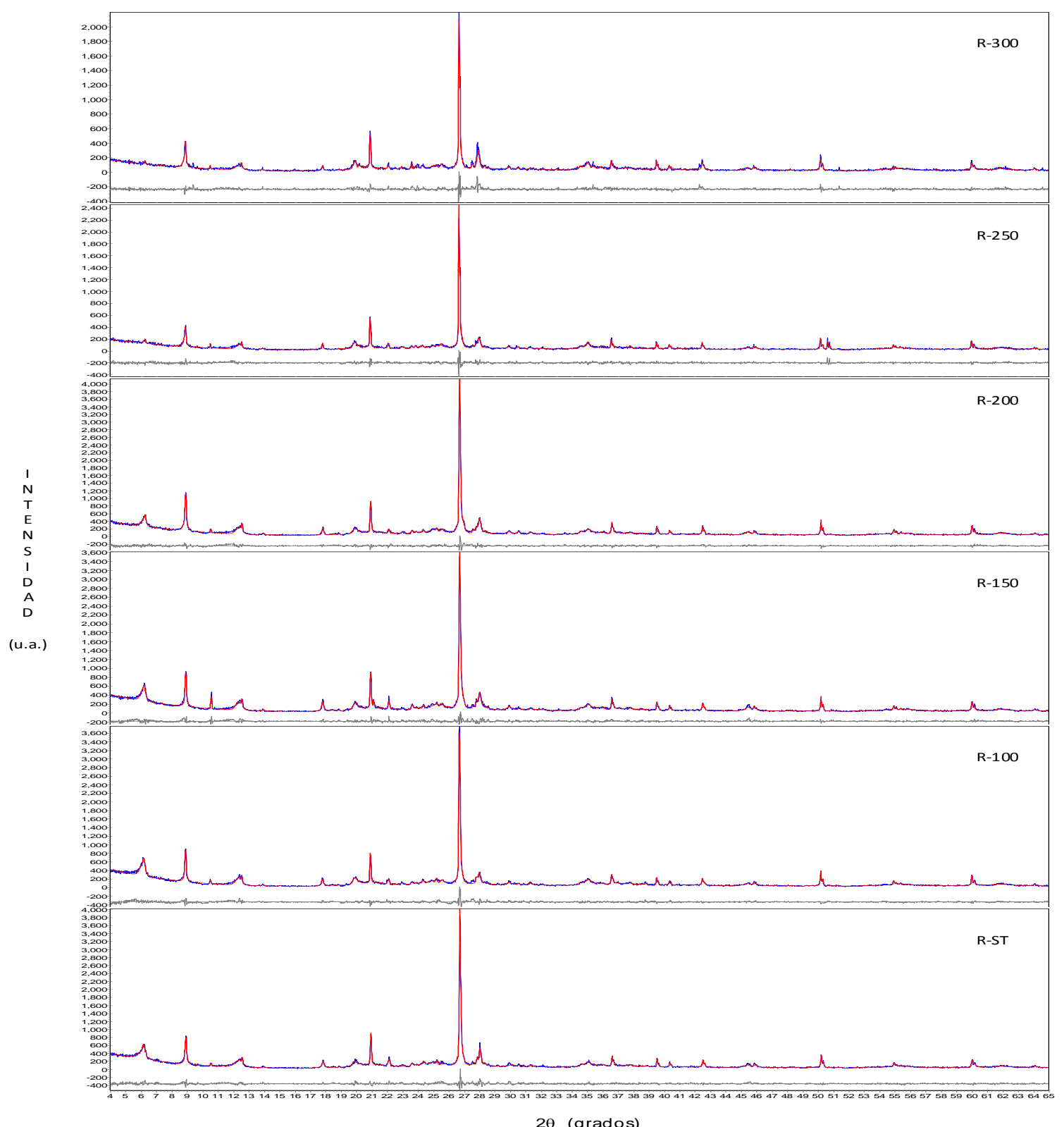

Figura 7: Refinamiento estructural por el método Rietveld de las muestras sin tratamiento térmico y las tratadas hasta $300^{\circ} \mathrm{C}$. 


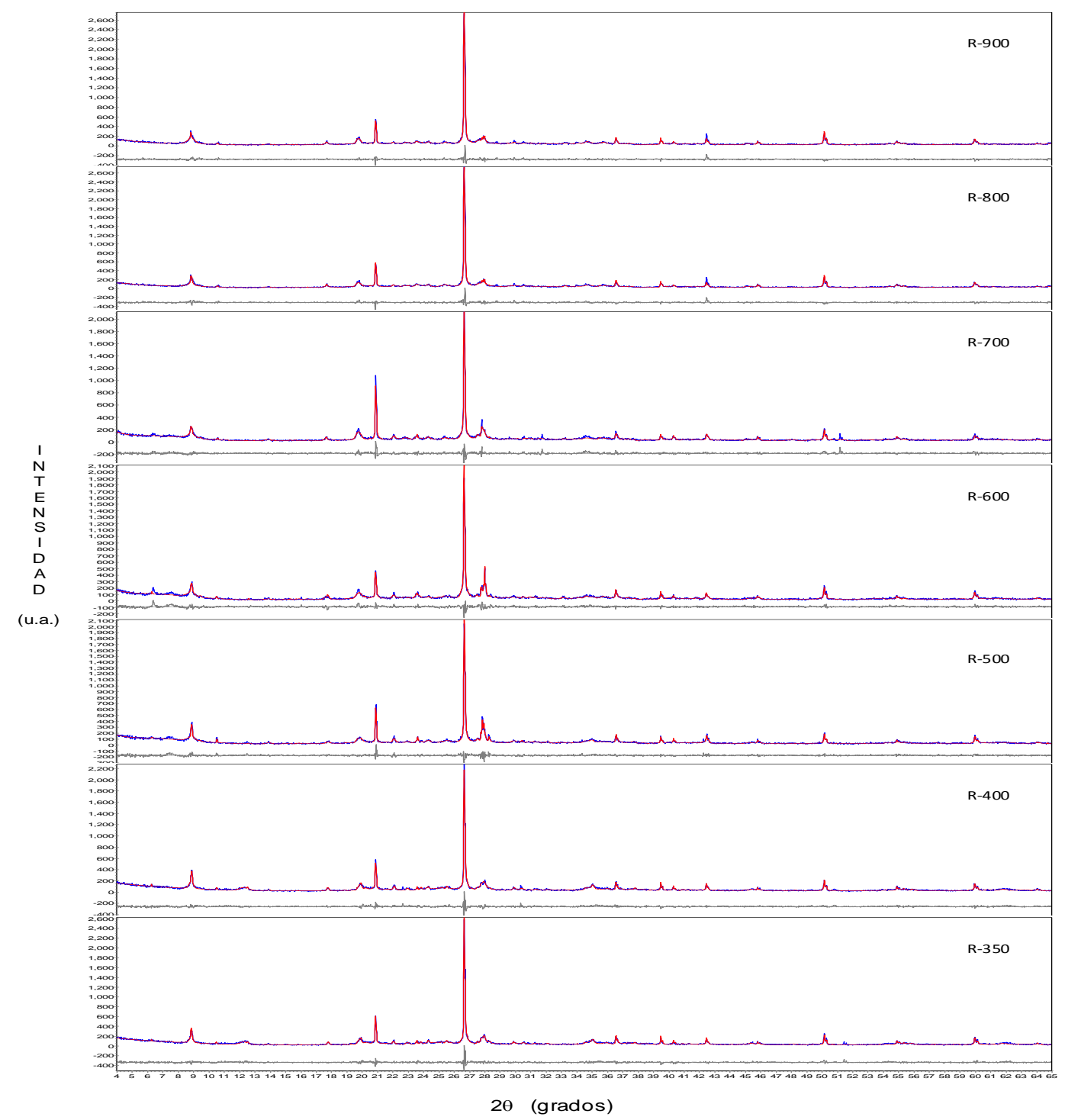

Figura 8: Refinamiento estructural por el método Rietveld de las muestras tratadas desde $350^{\circ} \mathrm{C}$ hasta $900^{\circ} \mathrm{C}$.

Los valores de los índices de ajuste $R_{\mathrm{wp}}$ y $R_{\text {exp }}$ del refinamiento son mostrados en la Tabla 1.

Tabla 1: Índices $R$ de refinamiento.

\begin{tabular}{c|c|c}
\hline \multirow{2}{*}{ Muestras } & \multicolumn{2}{|c}{ Índices de ajuste $(\%)$} \\
\cline { 2 - 3 } & $\mathbf{R}_{\mathbf{w p}}$ & $\mathbf{R}_{\exp }$ \\
\hline R-ST & 9,33 & 13,21 \\
\hline R-100 & 9,40 & 13,11 \\
\hline R-150 & 9,26 & 12,69 \\
\hline R-200 & 9,24 & 12,77 \\
\hline R-250 & 12,55 & 16,36 \\
\hline R-300 & 12,72 & 17,84 \\
\hline R-350 & 12,94 & 17,03 \\
\hline R-400 & 13,08 & 16,09 \\
\hline R-500 & 13,05 & 16,00 \\
\hline R-600 & 13,10 & 17,04 \\
\hline R-700 & 13,14 & 16,94 \\
\hline
\end{tabular}




\begin{tabular}{c|c|c}
\hline R-800 & 13,29 & 16,93 \\
\hline R-900 & 13,29 & 16,14 \\
\hline
\end{tabular}

Los cambios en la distancia interlaminar $d_{001}$ de la montmorillonita-Ca (arcilla altamente expansiva), debido al aumento de la temperatura, fueron determinados refinando los parámetros de la celda unitaria; los valores son mostrados en la Tabla 2, y caolinita (arcilla no-expansiva) donde también se incluye resultados para la caolinita (arcilla no-expansiva) y variaciones de la distancia $d_{002}$ de la vermiculita (arcilla moderadamente expansiva); la Figura 9 muestra gráficamente estos cambios.

Tabla 2: Valores de la distancia interlaminar (en $\mathrm{nm}$ ) de la montmorillonita-Ca, vermiculita y caolinita (ST:sin tratamiento térmico)..

\begin{tabular}{|c|c|c|c|c|c|c|c|c|c|}
\hline \multirow[t]{2}{*}{ FASES } & \multicolumn{9}{|c|}{ TEMPERATURA $\left({ }^{\circ} \mathbf{C}\right)$} \\
\hline & ST & 100 & 150 & 200 & 250 & 300 & 350 & 400 & 500 \\
\hline Montmorillonita-Ca & 1,500 & 1,465 & 1,460 & 1,450 & 1,006 & 1,003 & 1,003 & 1,003 & 1,002 \\
\hline Vermiculita & 2,921 & 2,902 & 2,894 & 2,877 & 2,877 & 2,872 & -- & -- & -- \\
\hline Caolinita & 0,743 & 0,742 & 0,742 & 0,742 & 0,740 & 0,740 & 0,743 & 0,744 & -- \\
\hline
\end{tabular}

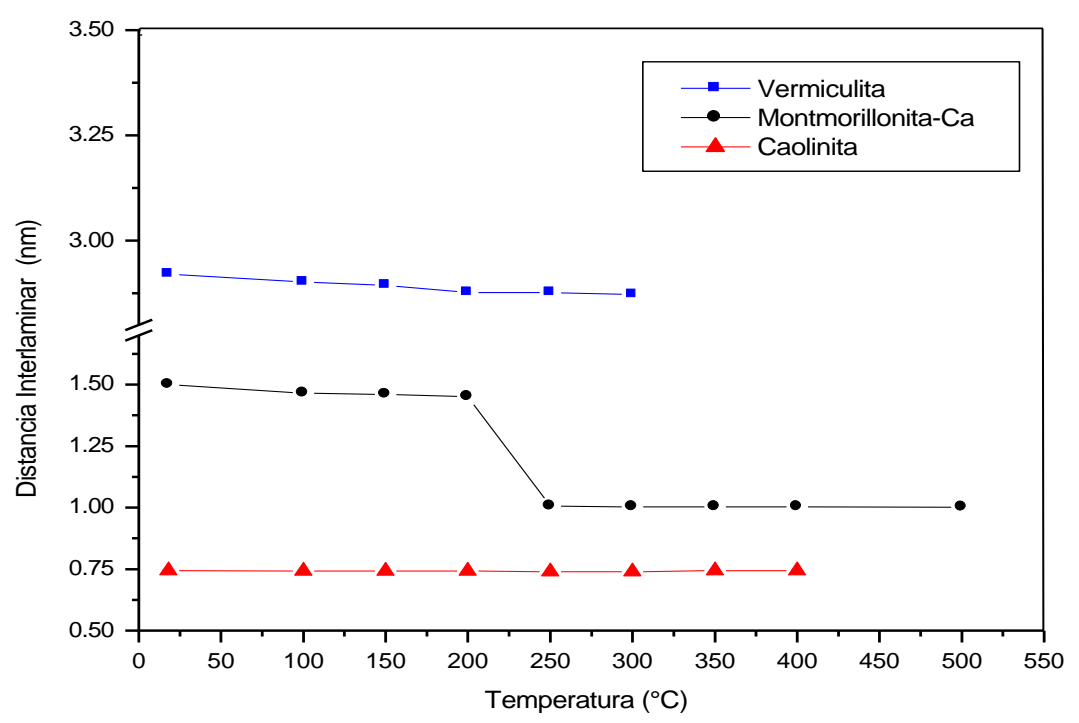

Figura 9: Efecto del aumento de temperatura sobre la distancia interlaminar de la montmorillonita-Ca (arcilla altamente expansiva), vermiculita (moderadamente expansiva) y caolinita (no-expansiva).

A $250^{\circ} \mathrm{C}$, la montmorillonita-Ca presenta una disminución drástica en su distancia interlaminar $d_{001}$, de $1.5 \mathrm{~nm}$ a $1.0 \mathrm{~nm}$, comportamiento característico de arcillas expansivas. Según la literatura, este tipo de montmorillonita tiene dos capas de agua en su región interlaminar [19]; con la cocción, el agua superficial y la de los poros es removida, así como la contenida en la región interlaminar. En tal sentido, asumimos que la disminución de $\sim 0.5 \mathrm{~nm}$ entre $200^{\circ} \mathrm{C}$ y $250^{\circ} \mathrm{C}$ se deba a la remoción de las dos capas de agua que ocupaban esta región, si consideramos que el tamaño de una molécula de agua es de $\sim 0.202 \mathrm{~nm}$. Para el caso de la vermiculita, la disminución de la distancia interlaminar es mucho menor ( $\sim 0.04 \mathrm{~nm})$, en tanto que la caolinita no presenta disminución significativa de este parámetro, pues la unión entre todas sus retículas es lo suficientemente firme para no permitir la penetración de moléculas de água [20], evitando con esto el hinchamiento y, consecuentemente, la contracción con la cocción.

La Tabla 3 presenta los porcentajes en peso de las fases de las muestras tratadas térmicamente hasta $500^{\circ} \mathrm{C}$, valores determinados por refinamiento. Como se observa, en todas las muestras hay mayor porcenta- 
je de arcilla no-expansiva (muscovita, illita, caolinita), en comparación con la expansiva (montmorillonita$\mathrm{Ca}$, vermiculita).

Tabla 3: Porcentaje en peso de las fases y su variación con el aumento de la temperatura (M: montmorillonita-Ca, V: vermiculita, Mu: muscovita, I: illita, Ca: caolinita, C: cuarzo, A: albita, R: riebeckita, Cl: chlorita, O: orthoclasa, An: anorthita).

\begin{tabular}{|c|c|c|c|c|c|c|c|c|c|c|c|}
\hline \multirow{2}{*}{$\begin{array}{c}\text { TEMP } \\
\left({ }^{\circ} \mathrm{C}\right)\end{array}$} & \multicolumn{11}{|c|}{ F A S E S } \\
\hline & $\mathbf{M}$ & $\mathbf{V}$ & $\mathbf{M u}$ & I & $\mathbf{C a}$ & $\mathrm{C}$ & $\mathbf{A}$ & $\mathbf{R}$ & $\mathrm{Cl}$ & $\mathbf{O}$ & An \\
\hline STT & 1,61 & 4,53 & 23,24 & 7,13 & 19,97 & 30,37 & 9,84 & 1,12 & 2,19 & -- & -- \\
\hline 100 & 1,93 & 2,65 & 27,43 & 6,73 & 15,41 & 32,83 & 8,58 & 1,86 & 2,57 & -- & -- \\
\hline 150 & 1,56 & 2,07 & 24,07 & 8,99 & 15,93 & 29,67 & 11,05 & 2,46 & 2,29 & 1,91 & -- \\
\hline 200 & 1,82 & 1,87 & 25,06 & 5,03 & 15,43 & 33,15 & 11,68 & 0,82 & 2,47 & 2,68 & -- \\
\hline 250 & 1,34 & 1,35 & 18,66 & 5,67 & 15,80 & 38,94 & 11,67 & 0,93 & 2,88 & 2,77 & -- \\
\hline 300 & 1,48 & 0,81 & 14,90 & 5,46 & 12,99 & 43,00 & 15,23 & 0,59 & 2,80 & 2,74 & -- \\
\hline 350 & 1,60 & -- & 12,33 & 6,17 & 12,63 & 46,57 & 15,37 & 0,70 & 2,27 & 2,36 & -- \\
\hline 400 & 2,17 & -- & 13,11 & 6,67 & 12,77 & 44,35 & 15,87 & 0,73 & 2,10 & 2,23 & -- \\
\hline 500 & 2,18 & -- & 13,89 & 7,26 & -- & 41,96 & 16,31 & 0,72 & 1,88 & 2,94 & 12,85 \\
\hline
\end{tabular}

Observamos que la montomorillonita-Ca es térmicamente más estable que la vermiculita y la caolinita; las últimas progresivamente disminuyen porcentualmente a medida que aumenta la temperatura. La vermiculita estructuralmente colapsa a $\sim 350^{\circ} \mathrm{C}$ y la caolinita lo hace a $\sim 500^{\circ} \mathrm{C}$, mientras que la montomorillonita-Ca estructuralmente se mantiene aún a esta temperatura, acompañando la formación de nuevas fases como la anorthita. A temperaturas menores $\left(\sim 150^{\circ} \mathrm{C}\right)$, se hace evidente la formación de orthoclasa.

\section{CONCLUSIONES}

Resultados de medidas de difracción de rayos-X mostraron una secuencia de modificaciones químicas y estructurales, como la deshidratación, descomposición y formación de nuevas fases, en las muestras de arcilla sometidas a tratamiento térmico. Los componentes de las muestras fueron determinados, identificándose montmorillonita-Ca, vermiculita, muscovita, caolinita, chlorita, illita, cuarzo y albita.

El refinamiento estructural reportó la presencia de un mayor porcentaje de arcilla no-expansiva (muscovita, illita, caolinita), en comparación con la expansiva (montmorillonita-Ca, vermiculita). A $250^{\circ} \mathrm{C}$, la montmorillonita-Ca presenta una disminución drástica en su distancia interlaminar, de $1.5 \mathrm{~nm}$ a $1.0 \mathrm{~nm}$, debido a la remoción del agua superficial y de los poros, así como de la contenida en la región interlaminar. En la vermiculita, esta disminución es mucho menor $(\sim 0.04 \mathrm{~nm})$, en tanto que la caolinita no presenta disminución significativa en el valor de este parámetro. Por otro lado, la montomorillonita-Ca presenta mayor estabilidad térmica que la vermiculita y la caolinita: la vermiculita estructuralmente colapsa a $\sim 350^{\circ} \mathrm{C}$, en tanto que la caolinita lo hace a $\sim 500^{\circ} \mathrm{C}$, mientras que la montmorillonita-Ca se mantiene a esta temperatura. Simultáneamente se evidencia la formación de nuevas fases, como la ortoclasa y la anorthita.

La difracción de rayos- $\mathrm{X}$ y el método de Rietveld han resultado ser sumamente útiles para el entendimiento de los cambios estructurales de las muestras investigadas en el presente trabajo, constituyéndose en importantes herramientas para monitorear estos cambios en sistemas desordenados como las arcillas.

\section{AGRADECIMIENTOS}

Nuestro agradecimiento al Sr. Gerásimo Sosa Alache, ceramista de Chulucanas de prestigio internacional, por proporcionarnos las muestras y por transmitirnos su valiosa experiencia profesional. Asimismo, agradecemos al Vice-Rectorado de Investigación de la Universidad Nacional Mayor de San Marcos por el soporte financiero. esta seção podem ser dados os créditos aos suportes recebidos no trabalho. 


\section{BIBLIOGRAFÍA}

[1] BRAY, H.J., REDFERN, S.A.T., CLARK, S.M., "The kinetics of dehydration in Ca-montmorillonite: an in situ X-ray diffraction study”, Mineralogical Magazine, v. 62, n. 5, pp. 647-656, 1998.

[2] MOORE, D.M., X-Ray Diffraction and the identification and analysis of clay minerals, Oxford University Press, New York, USA, 1997.

[3] HÄUSLER, W. "Firing of Clays Studied by X-ray Diffraction and Mössbauer Spectroscopy", Hyperfine Interactions, v. 154, pp. 121-141, 2004.

[4] ZEBALlOS-VELÁSQUEZ, E. L., TRUJILlO, A. L., MEJÍA, M.E., et al, "Evaluación de la estabilidad térmica de arcillas de Chulucanas por Difracción de Rayos-X y Método de Rietveld", Int. Electron J. Nanoc. Moletrón, v. 10, n. 2, pp. 1899-1914, 2012.

[5] ZEBALlOS-VELÁSQUEZ, E. L., TRUJILlO, A. L., MEJÍA, M.E., et al, "Estudio de las reacciones térmicas de arcillas de Chulucanas por Espectroscopia Mössbauer", Int. Electron J. Nanoc. Moletrón. v. 10, n. 2, pp. 1945-1958, 2012.

[6] ZEBAllos-VElÁSQUEZ, E. L., MIÑANO, M.V., MELERO, P.C.,"Caracterización de arcillas de Nazca por difracción de rayos X y refinamiento estructural por el Método de Rietveld”, Int. Electron J. Nanoc. Moletrón, v. 11, n. 1, pp 2001-2018, 2013.

[7] TRUJILLO, A.L., ZEBALLOS-VELÁSQUEZ, E.L., MIÑANO, M.V., et al.,“Análisis del efecto térmico en arcillas de Nazca por Espectroscopia Mössbauer", Int. Electron J. Nanoc. Moletrón, v. 11, n. 1, pp 2041-2056, 2013.

[8] ZEBALlOS-VELÁSQUEZ, E.L., MEJÍA, M., TRUJILLO, A., et al., "Estudio de la estabilidad térmica de arcillas por Difracción de rayos-X y refinamiento Rietveld". In: II Congreso Latinoamericano de Arqueometría, Oct. 2009.

[9] ZEBALLOS-VELÁSQUEZ, E.L., MEJÍA, M., TRUJILLO, A., “Análisis del efecto térmico en arcillas por Difracción de Rayos-X y Refinamiento Rietveld: determinación cuantitativa de fases”. In: Encuentro Científico Internacional 2009 de invierno, ECI-2009i, Ag. 2009.

[10] TRUJILlO, A.L., MEJÍA, M.E., ZEBALLOS-VELÁSQUEZ, E.L., et al., "Evaluación del efecto de la temperatura en arcillas de Chulucanas usando Espectroscopia Mössbauer y Fluorescencia de Rayos-X". (sometido a la Revista CIENCIA, Maracaibo, Venezuela).

[11] BISH, D. L., "Studies of Clays and Clay Minerals using X-Ray Powder Diffraction and the Rietveld Method". In: CMS workshop lectures, v.5, Computer Applications to X-Ray Powder Diffraction analysis of Clay Minerals, Reynolds, R.C. and Walker, J.R. Eds., CMS, Boulder, 80-121, 1993.

[12] BONETTO, R.D., ZALBA, P.E., CONCONI, M.S., et al., "The Rietveld method applied to quantitative phase analysis of minerals containing disordered structures”. Revista Geológica de Chile v. 30, n. 1, pp. 103-115, 2003.

[13] YOUNG, R.A, LARSON, A.C., PAIVA-SANTOS, C.O., "User's Guide to Program DBWS-9807a for Rietveld Analysis of X-ray and Neutron Powder Diffraction Patterns with a 'PC' and various other Computers", In: School of Physics Georgia Institut of Technology Atlanta, GA 30332, 1999.

[14] YOUNG, R.A., The Rietveld Method. International Union Crystallography, Oxford University Press. New York, USA, 1993.

[15] RIETVELD, H.M. “A profile refinement method for nuclear and magnetic structures”, Journal of Applied Crystallography, v. 2, pp. 65-71, 1969.

[16] TOPAS User's Manual, BRUKER AXS GmbH, Karlsruhe, West Germany.

[17] DIFFRACplus, TOPAS/TOPAS R/TOPAS P., Version 3.0, Technical Reference, BRUKER AXS GmbH, Karlsruhe, West Germany.

[18] GERASIMO2000'S PHOTOSTREAM, http://www.flickr.com/photos/gerasimo2000/page11/. Accesado en Julio de 2012.

[19] ODRIOZOLA, G., AGUILAR, J.F. "Stability of Ca-montmorillonite hydrates: A computer simulation study”, J. Chem. Phys., v.123, n.17, 2005. http://dx.doi.org/10.1063/1.2087447. Accesado en Marzo de 2013.

[20] JUÁREZ, E., RICO, A., Mecánica de suelos, Limusa, México, 2005. 